\title{
Monte Carlo sampling of particle-hole creation transitions in a model of pre-equilibrium reactions
}

\author{
Daniel Farias Mega*t \\ Instituto Tecnológico de Aeronáutica, São José dos Campos SP Brasil \\ E-mail: danielfmeg@gmail.com

\section{B. V. Carlson} \\ Instituto Tecnológico de Aeronáutica, São José dos Campos SP Brasil \\ E-mail: bretteita.br
}

\begin{abstract}
Pre-equilibrium processes play an important role in nucleon-induced reactions above about 10 $\mathrm{MeV}$. Models that describe such reactions were developed long ago. One of the more recent models of such processes, the Double Differential Hybrid Monte Carlo Simulation (DDHMS) of M. Blann and M. B. Chadwick, [1] follows the cascade of particle-hole pairs created by the incident nucleon and subsequent excited particles and holes until all excited particles are either emitted or, together with the excited holes, equilibrated. The model has the advantage over older models of permitting the emission of several nucleons and of not requiring doubtful hypotheses concerning statistical equilibrium [2] In this work, we analyze the momenta and angles of the resulting particles and hole relative to the momentum and direction of the nucleon inducing the transition.
\end{abstract}

XXXIV edition of the Brazilian Workshop on Nuclear Physics,

5-10 June 2011

Foz de Iguaçu PR Brasil

\footnotetext{
${ }^{*}$ Speaker.

${ }^{\dagger}$ DFM is supported by CAPES. BVC acknowledges support from FAPESP and the CNPq.
} 


\section{Introduction}

Nuclear reactions were initially classified as either direct or equilibrium processes. In a direct nucleon-induced reaction, the incident nucleon interacts with a target nucleus on a time scale of the order of the time it takes to pass by the target and often excites collective degrees of freedom of the target. A nucleon-induced equilibrium process can be initiated by a direct reaction, but usually involves the absorption of the incident nucleon followed, after a relatively long time, by the emission of other nucleons, composite particles and $\gamma$ rays. This decay process is well described by the statistical theory of nuclear reactions, which assumes that the nucleus reaches internal equilibrium before decaying.

However experimental measurements show that reactions intermediate between the two also occur, in which particles are emitted before complete equilibrium is reached. Such reactions are called pre-equilíbrium reactions and have been studied extensively. The first model of preequilibrium reactions, the exciton model, was formulated by Griffin in 1966 [3]. This semi-classical model classified nuclear excitations as particles above and holes below the Fermi energy, jointly called excitons. It sought to describe the formation and decay of the nuceus resulting from a nucleon-induced reaction at each stage, until the nucleus reaches equilibrium. The exciton model was extended by Blann [4], by introducing the possibility of emitting charged particles, and by Williams [5], who extended the densities of excitons states and of transitions by including a correction for Pauli blocking and extending the densities to two types of fermions. Another semi-classical model frequently used to describe preequilibrium reactions is the hybrid model, proposed by Blann in 1971 [6]. There, he used the same basic ideas of Griffin to describe the intermediate states together with the description of particle emission and transitions proposed by Harp, Miller and Berne [7].

After serious criticisms of the statistical hypotheses used in the exciton and hybrid model were put forth by Bisplinghoff [8], Blann proposed an alternative, which he called the hybrid Monte Carlo simulation (HMS) model [9]. This uses the basic emission and transition rates of the hybrid model but makes no assumptions concerning the statistical equilibrium of the excited holes and particles. Rather, each of these is treated as an independent excitation, with subsequent emissions and rescatterings followed using a Monte Carlo simulation. An additional advantage of the HMS model compared to earlier models is that it easily permits multiple emissions from the precompound nucleus. Two years later, Blann and Chadwick, generalized the model to the DDHMS (double differential hybrid Monte Carlo simulation) model by including the calculation of angular distributions based on the work of Chadwick and Oblozinský[10, 11]. Here, we propose a Monte Carlo method for calculating the exact energy-angular distributions of the particles and holes of the DDHMS transition rates.

\section{Monte Carlo sampling of $1 p \rightarrow 2 p 1 h$ transitions and the $1 p \rightarrow 2 p 1 h$ transition density}

Up to a constant multiplicative factor, we can define the $1 p \rightarrow 2 p 1 h$ transition density by 


$$
\begin{aligned}
\rho_{1 p \rightarrow 2 p 1 h}\left(\vec{p}_{1}\right)= & \frac{a}{(2 \pi \hbar)^{6}} \int \delta\left(\vec{p}_{1}+\vec{p}_{2}-\vec{p}_{3}-\vec{p}_{4}\right) \delta\left(\frac{p_{1}^{2}}{2 m}+\frac{p_{2}^{2}}{2 m}-\frac{p_{3}^{2}}{2 m}-\frac{p_{4}^{2}}{2 m}\right) \\
& \times \theta\left(p_{F 2}-\left|\vec{p}_{2}\right|\right) d^{3} p_{2} \theta\left(\left|\vec{p}_{3}\right|-p_{F 1}\right) d^{3} p_{3} \theta\left(\left|\vec{p}_{4}\right|-p_{F 2}\right) d^{3} p_{4},
\end{aligned}
$$

We identify the incident particle 1 with the final particle 3 and the incident particle (final hole) 2 with the final particle 4, by giving each pair the same Fermi momentum. This expression enforces momentum and energy conservation and limits the particle and hole momenta to

$$
\left|\vec{p}_{1}\right| \geq p_{F 1}, \quad\left|\vec{p}_{2}\right| \leq p_{F 2}, \quad\left|\vec{p}_{3}\right| \geq p_{F 1} \text { e }\left|\vec{p}_{4}\right| \geq p_{F 2}
$$

To sample the distributions of the final momenta $\vec{p}_{2}, \vec{p}_{3}$ and $\vec{p}_{4}$, we will first choose the magnitude of the hole momentum, $p_{2}$, produced in the reaction. We next choose the angle $\theta_{12}$ between the initial particle and hole momenta. We then choose the magnitude of the momentum of particle 3 generated in the reaction, $p_{3}$. Finally we determine the momentum of the particle, $p_{4}$, using energy conservation and the angle between particles 3 and $4, \theta_{34}$, using conservation of the magnitude of the total momentum.

To use this procedure to determine the momenta, we must transform the integral 2.1 defining the transitions densities so that it makes use of the same variables. Making all necessary changes, we find

$$
\rho_{1 p \rightarrow 2 p 1 h}\left(\vec{p}_{1}\right)=\frac{a}{(2 \pi)^{4} \hbar^{6}} \frac{m}{p_{1}} \int \theta\left(p_{F 2}-p_{2}\right)\left(p_{1}^{2}+p_{2}^{2}-p_{F 1}^{2}-p_{F 2}^{2}\right) p_{2}^{2} d p_{2}
$$

which results in

$$
\rho_{1 p \rightarrow 2 p 1 h}\left(\vec{p}_{1}\right)=\frac{a}{(2 \pi)^{4} \hbar^{6}} \frac{m}{p_{1}}\left[\left(p_{1}^{2}-p_{F 1}^{2}-p_{F 2}^{2}\right) \frac{p_{2}^{3}}{3}+\frac{p_{2}^{5}}{5}\right]_{p_{2}=p_{2 m i n}}^{p_{2}=p_{F 2}}
$$

where $p_{2 \min }^{2}=\max \left(0, p_{F 1}^{2}+p_{F 2}^{2}-p_{1}^{2}\right)$. This furnishes

$$
\begin{aligned}
\rho_{1 p \rightarrow 2 p 1 h}\left(\vec{p}_{1}\right) & =\frac{a m}{2(2 \pi)^{4} \hbar^{6} p_{1}}\left[\left(p_{1}^{2}-p_{F 1}^{2}-\frac{2 p_{F 2}^{2}}{5}\right) \frac{p_{F 2}^{3}}{3}+\frac{p_{2 \min }^{5}}{15}\right] \\
& =\left\{\begin{array}{cc}
\frac{a m}{2(2 \pi)^{4} \hbar^{6} p_{1}}\left(p_{1}^{2}-p_{F 1}^{2}-\frac{2 p_{F 2}^{2}}{5}\right) \frac{p_{F 2}^{3}}{3} & p_{1}^{2} \geq p_{F 1}^{2}+p_{F 2}^{2} \\
\frac{a m}{(2 \pi)^{4} \hbar^{6} p_{1}}\left(p_{1}^{2}-p_{F 1}^{2}\right)\left(p_{1}^{2}-p_{F 1}^{2}+\frac{p_{F 2}^{2}}{2}\right) \frac{p_{F 2}}{15} & p_{1}^{2} \leq p_{F 1}^{2}+p_{F 2}^{2} .
\end{array}\right.
\end{aligned}
$$

We are now ready to determine the momenta $\vec{p}_{2}, \vec{p}_{3}$ and $\vec{p}_{4}$ by Monte Carlo sampling. We first choose the magnitude of the hole momentum, $p_{2}$, which is limited by

$$
p_{2 \min }^{2} \leq p_{2}^{2} \leq p_{F 2}^{2}
$$

The value of $p_{2}$ is determined so that the frequency of a given value will be in agreement with the integral of its distribution. For a random number $0 \leq x \leq 1$, we define the corresponding value of $p_{2}$ to be 


$$
x=\frac{\left[\left(p_{1}^{2}-p_{F 1}^{2}-p_{F 2}^{2}\right) \frac{p_{2}^{3}}{3}+\frac{p_{2}^{5}}{5}+\frac{2 p_{2 \min }^{5}}{15}\right]}{\left[\left(p_{1}^{2}-p_{F 1}^{2}-\frac{2 p_{F 2}^{2}}{5}\right) \frac{p_{F 2}^{3}}{3}+\frac{2 p_{2 \min }^{5}}{15}\right]} .
$$

We next choose the angle $\theta_{12}$ between the initial particle and the hole momenta, which is unrestricted in value,

$$
-1 \leq \cos \theta_{12} \leq 1
$$

In this case, we associate a value of $\cos \theta_{12}$ to a random value $0 \leq x \leq 1$ by requiring that

$$
x=\frac{\sqrt{p_{1}^{2}+p_{2}^{2}+2 p_{1} p_{2} \cos \theta_{12}}-\left|p_{1}-p_{2}\right|}{\left|p_{1}+p_{2}\right|-\left|p_{1}-p_{2}\right|}
$$

The next choice is that of the magnitude of the final particle momentum, $p_{3}$, which is limited by

$$
p_{F 1}^{2} \leq p_{3}^{2} \leq p_{1}^{2}+p_{2}^{2}-p_{F 2}^{2} .
$$

The value of $p_{3}$ corresponding to a random value $0 \leq x \leq 1$ is defined by

$$
x=\frac{p_{3}^{2}-p_{F 1}^{2}}{p_{1}^{2}+p_{2}^{2}-p_{F 1}^{2}-p_{F 2}^{2}} .
$$

The value of $p_{4}$ is then determined by energy conservation and the angle between the final momenta $\theta_{34}$ is determined by conservation of the magnitude of the total momentum.

\section{Monte Carlo sampling of $1 h \rightarrow 1 p 2 h$ transitions and the $1 h \rightarrow 1 p 2 h$ transition density}

Sampling the distributions of the final momenta $\vec{p}_{2}, \vec{p}_{3}$ and $\vec{p}_{4}$ of the $1 h \rightarrow 1 p 2 h$ transiton density by Monte Carlo sampling is very similar to that of the $1 p \rightarrow 2 p 1 h$ distribution. In this case, the density is proportional to,

$$
\begin{aligned}
\rho_{1 h \rightarrow 1 p 2 h}\left(\vec{p}_{1}\right)= & \frac{a}{(2 \pi \hbar)^{6}} \int \delta\left(\vec{p}_{1}+\vec{p}_{2}-\vec{p}_{3}-\vec{p}_{4}\right) \delta\left(\frac{p_{1}^{2}}{2 m}+\frac{p_{2}^{2}}{2 m}-\frac{p_{3}^{2}}{2 m}+\frac{p_{4}^{2}}{2 m}\right) \\
& \times \theta\left(\left|\vec{p}_{2}\right|-p_{F 2}\right) d^{3} p_{2} \theta\left(p_{F 1}-\left|\vec{p}_{3}\right|\right) d^{3} p_{3} \theta\left(p_{F 2}-\left|\vec{p}_{4}\right|\right) d^{3} p_{4},
\end{aligned}
$$

with the limits on the particle and hole momenta now being just the opposite of the previous case,

$$
\left|\vec{p}_{1}\right| \leq p_{F 1}, \quad\left|\vec{p}_{2}\right| \geq p_{F 2}, \quad\left|\vec{p}_{3}\right| \leq p_{F 1} \quad e \quad\left|\vec{p}_{4}\right| \leq p_{F 2} .
$$

Using the same procedure used above the integral 3.1 can be reduced to

$$
\rho_{1 h \rightarrow 1 p 2 h}\left(\vec{p}_{1}\right)=\frac{a m}{(2 \pi)^{4} \hbar^{6} p_{1}} \int \theta\left(p_{2}-p_{F 2}\right)\left(p_{F 1}^{2}+p_{F 2}^{2}-p_{1}^{2}-p_{2}^{2}\right) p_{2} d p_{2}
$$

The integral over $p_{2}$ furnishes 


$$
\rho_{1 h \rightarrow 1 p 2 h}\left(\vec{p}_{1}\right)=\frac{a m}{4(2 \pi)^{4} \hbar^{6}}\left(p_{F 1}^{2}-p_{1}^{2}\right)^{2} .
$$

The Monte Carlo sampling can be performed much as before. The only differences here are in the distributions of the variables $p_{2}$ e $p_{3}$.

Here, the magnitude of the hole, $p_{2}$ is limited by

$$
p_{F 2}^{2} \leq p_{2}^{2} \leq p_{F 1}^{2}+p_{F 2}^{2}-p_{1}^{2}
$$

For a random number $0 \leq x \leq 1$, we define the corresponding value of $p_{2}$ as

$$
x=\frac{2\left[\left(p_{F 1}^{2}+p_{F 2}^{2}-p_{1}^{2}-\frac{p_{2}^{2}}{2}\right) p_{2}^{2}-\left(p_{F 1}^{2}+\frac{p_{F 2}^{2}}{2}-p_{1}^{2}\right) p_{F 2}^{2}\right]}{\left(p_{F 1}^{2}-p_{1}^{2}\right)^{2}} .
$$

The angle $\theta_{12}$ between the initial particle and the hole momenta can be chosen as before.

The magnitude of the final momentum, $p_{3}$, is limited by

$$
p_{1}^{2}+p_{2}^{2}-p_{F 2}^{2} \leq p_{3}^{2} \leq p_{F 1}^{2},
$$

with the value of $p_{3}$ corresponding to the random value $0 \leq x \leq 1$ defined by

$$
x=\frac{p_{3}^{2}+p_{F 1}^{2}-p_{1}^{2}-P_{2}^{2}}{p_{F 1}^{2}+p_{F 2}^{2}-p_{1}^{2}-p_{2}^{2}}
$$

The remaining momentum $p_{4}$ and angles are determined as in the $1 p \rightarrow 2 p 1 h$ case

\section{Results}

The distributions of particles and holes were obtained using a small Fortran code. Results for two values of the incident energy are shown here: one below the Fermi energy, at $15 \mathrm{MeV}$, and one above it, at $133 \mathrm{MeV}$. We take the Fermi momentum to be $33 \mathrm{MeV}$. Note that we have defined both particle and hole energies with respect to the zero momentum/energy state. Hole energies and momenta are thus actually those of the unoccupied particle states and not the values conventionally used for holes. Particle energies include the Fermi energy.

\subsection{Incident Momentum of $15 \mathrm{MeV}: 1 h \rightarrow 1 p 2 h$.}

Below we show the distributions of the particle and either of the holes produced in a $1 h \rightarrow 1 p 2 h$ transition induced by a $15 \mathrm{MeV}$ energy hole. The particle distribution is peaked at backward angles and at the Fermi momentum while the hole distributions peaks at forward angles and at the Fermi momentum. 

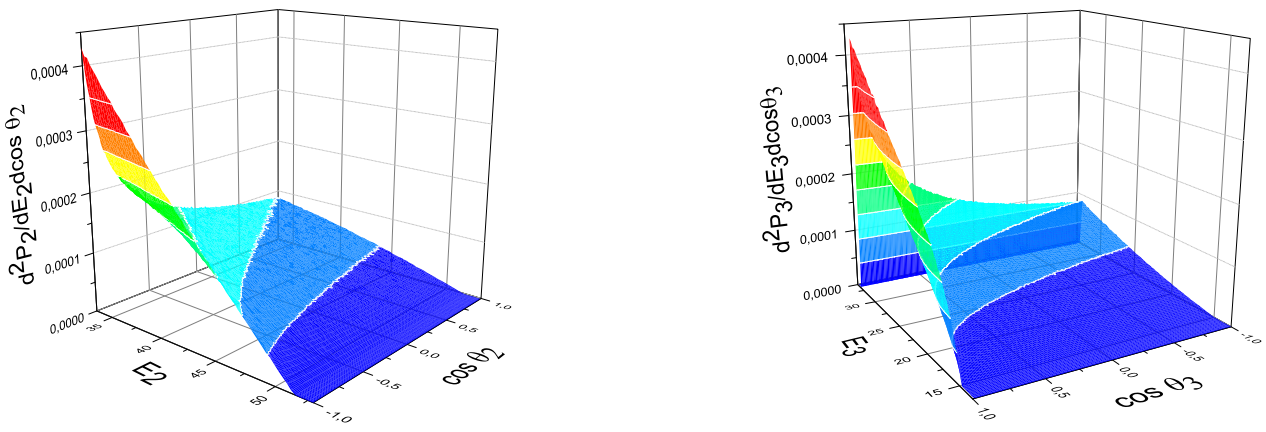

Figure 1: Distribution of the particle and the holes generated in a $1 h \rightarrow 1 p 2 h$ transition.

\subsection{Incident Energy of $133 \mathrm{MeV}: 1 p \rightarrow 2 p 1 h$}

Here we display the distributions of the hole and either of the two particles produced in a $1 p \rightarrow 2 p 1 h$ transition induced by a $133 \mathrm{MeV}$ energy particle. Here the hole momentum distribution peaks at backward angles and at the Fermi energy while the particle distribution peaks at extreme forward angles and values of the momentum close to the initial one.
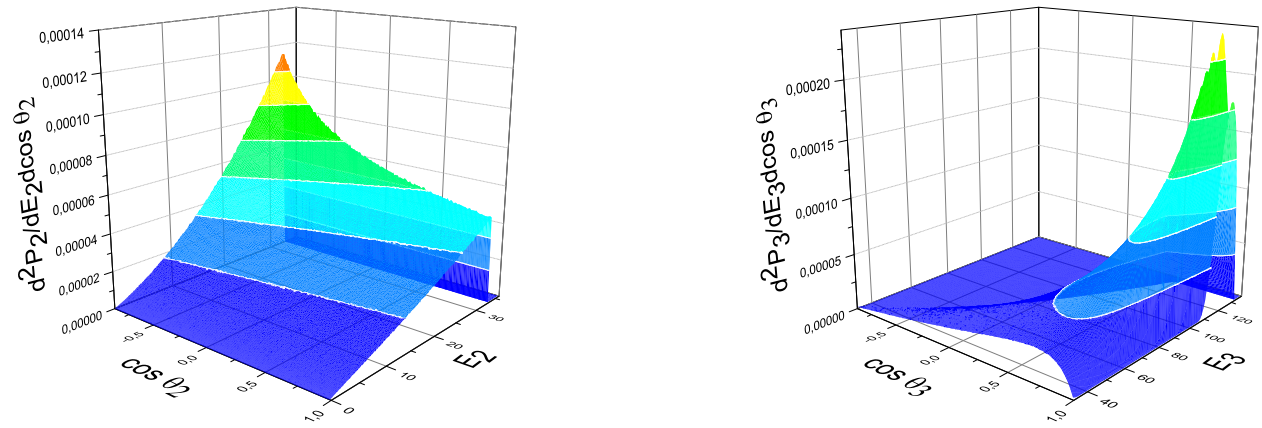

Figure 2: Distribution of the hole and the particles generated in a $1 p \rightarrow 2 p 1 h$ transition.

\section{Conclusion}

We have developed a Monte Carlo algorithm for sampling the $2 p 1 h$, energy-angular distribution resulting from a transition induced by a particle in nuclear matter, as well as the $1 p 2 h$ energyangular distribution resulting from a transition induced by a hole. Our algorithm furnishes a fast means of sampling the exact energy-angular distributions of the transition rates of the DDHMS model. We plan to perform DDHMS calulations using these in the near future.

\section{References}

[1] M. Blann and M. B. Chadwick, New precompound decay model: Angular distributions, Phys. Rev. C 57 (1998) 233. 
[2] C. A Soares Pompéia and B. V. Carlson, Configuration mixing in pre-equilibrium reactions, Phys. Rev. C 74 (2006) 054609.

[3] J. J. Griffin, Statistical model of intermediate structure, Phys. Rev. Lett. 17 (1966) 57.

[4] M. Blann, Extensions of Griffint's model for medium-energy nuclear reactions, Phys. Rev. 21 (1968) 1357.

[5] F. C. Williams, Particle-hole state density in the uniform spacing model, Nucl. Phys A166 (1971) 231.

[6] M. Blann, Hybrid model for pre-equilibrium decay in nuclear reactions, Phys. Rev. Lett. 27 (1971) 337.

[7] G. D. Harp, J. M. Miller and B. J. Berne, Attainment of statistical equilibrium in excited nuclei Phys. Rev. 165 (1968) 1166.

[8] J. Bisplinghoff, Configuration mixing in preequilibrium reactions: A new look at the hybrid-exciton controversy Phys. Rev. C 33 (1986) 1569.

[9] M. Blann, New precompound decay model, Phys. Rev. C 54 (1996) 1341.

[10] M. B. Chadwick and P. Oblozinský, Particle-hole state densities with linear momentum and angular distributions in preequilibrium reactions, Phys, Rev. C 46 (1992) 2028.

[11] M. B. Chadwick and P. Oblozinský, Continuum angular distributions in preequilibrium nuclear reactions: Physical basis for Kalbach systematics, Phys, Rev. C 50 (1994) 2490. 